\title{
Die sogenannte Feminisierung der Medizin - unter spezieller Fokussierung auf die Nachwuchsgewinnung in Unfallchirurgie und Orthopädie
}

\author{
The So-Called Feminisation of Medicine - With a Special Focus on the
}

Recruitment of Young Surgeons for Trauma Surgery and Orthopaedics

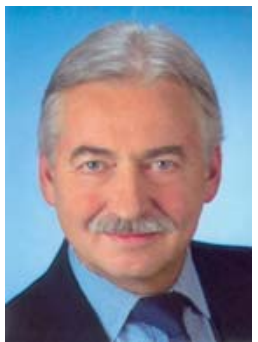

K. Weise

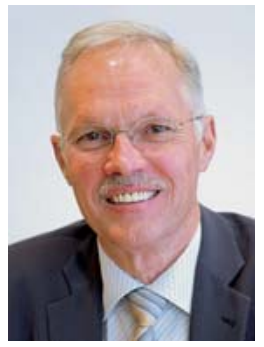

F. U. Niethard

Bibliografie

DOI http://dx.doi.org/

10.1055/s-0030-1250035

Z Orthop Unfall 2010; 148: 273-

275 @ Georg Thieme Verlag KG Stuttgart · New York .

ISSN 1864-6697

\section{Korrespondenzadressen}

Prof. Dr. med. Kuno Weise BG-Unfallklinik

Schnarrenbergstraße 95

72076 Tübingen

Tel.: 07071/606-1001

Fax: 07071/606-1002

weise@bgu-tuebingen.de

Prof. Dr. med. Fritz U. Niethard Orthopädische Klinik Universitätsklinik der RWTH Aachen

Pauwelsstraße 30

52074 Aachen

Tel.: 0241/8 08-94 10

Fax: 0241/808-2453

funiethard@

orthopaedie-aachen.de
Feminisierung = Verweiblichung: Gesamtheit der körperlichen und psychischen Veränderungen beim Mann...

Meyers Neues Lexikon

Schon alleine die semantische Bewertung dieses Begriffs bemüßigt mich zu uneingeschränkter Zustimmung hinsichtlich der Ausführungen von Frau Bühren, dass dessen Verwendung im Kontext zu Negativentwicklungen in der Medizin als obsolet anzusehen ist [4]. Frau Bühren thematisiert in ihrer Eröffnungsrede anlässlich des 31. Wissenschaftlichen Kongresses des Deutschen Ärztinnenbunds vom 11. bis 13.09.2009 in Leipzig, der unter dem Motto „Ärztin Macht Prävention - Generation Gesundheit - Investition in die Zukunft" stand, die Frage, ob „Ärztinnen Sündenböcke, Lückenbüßerinnen - oder doch die Zukunftsperspektive für die Medizin?" seien [3]. Zur derzeitigen Realität der Diskussion um die sog. „Feminisierung" führt sie aus, dass wir nunmehr einen Normalisierungsprozess weg von der Maskulinisierung der Medizin hin zur Erlangung eines überfälligen und auch notwendigen zahlenmäßigen Gleichgewichts von Ärztinnen und Ärzten erleben würden. Es sei darüber hinaus Vorsorge zu treffen, Ärztinnen nicht länger als Sündenböcke dafür anzusehen, dass einem in vieler Hinsicht immer unattraktiver werdenden Beruf in Zukunft der Nachwuchs fehlt.

Lindhorst und Tempka [10] beleuchten 2005 in einem Aufsatz unter dem Titel „Unruhige Zeiten an deutschen Kliniken“ u.a. auch den Aspekt der zunehmenden Anzahl von Medizinstudentinnen. Sozialwissenschaftlich sei dies ein Indikator für eine schlechte finanzielle Entlohnung bei gleichzeitig hohem sozialem Engagement in diesem Beruf. Darüber hinaus halten die Autoren fest, dass die Feminisierung der Medizin erhebliche Konsequenzen für die Patientenversorgung habe. Ärztinnen müssten in Zukunft, noch mehr als bisher, fester Bestandteil auch in den sog. Männerdomänen der Medizin sein, um die Patientenversorgung aufrecht erhalten zu können.

Botzlar [1] geht 2009 in einem Vortrag zum Thema „Motivationskrise junger Ärztinnen und Ärzte" auch auf den Umstand ein, dass die einem Arbeitgeber (!) zur Verfügung stehende Lebensarbeitsleistung von 3 Frauen derjenigen von 2 Männern entspräche... Es werde behauptet, dass der steigende Anteil von weiblichen Medizinstu- dierenden auf die sinkende ökonomische Attraktivität des Arztberufs zurückzuführen sei; männliche Studierende würden sich Fächern mit besseren Karrierechancen zuwenden.

Nüssler [12] führt 2009 zum Thema „Attraktivitätsverlust der Chirurgie - wie lässt sich gegensteuern?" aus, dass sich das Nachwuchsproblem noch weiter verschärfen werde, da unter weiblichen Medizinstudenten, die inzwischen die Mehrheit, nämlich mehr als 60\% der Studierenden ausmachten, die Chirurgie als ganz besonders unattraktiv gelte... Die Balance zwischen Arbeit und Privatleben, etwa mit Partnern und Kindern, gewinne übrigens keineswegs nur für Frauen, sondern auch für Männer zunehmend an Bedeutung. Leschber [7] berichtet 2007 über das Projekt „FiT - Frauen in der Thoraxchirurgie“ und stellt angesichts des hohen Anteils weiblicher Studierender im Unterschied zu Nüssler fest, dass immer mehr Frauen in ein chirurgisches Fach strebten, denn die Begeisterung für die Chirurgie unterscheide sich bei Männern und Frauen initial nicht, ebenso wenig wie handwerkliche Geschicklichkeit, Einsatzfreude und körperliche Belastbarkeit. Gleichwohl beeinflussten verschiedene Faktoren die Entscheidung, dass Frauen eine chirurgische Ausbildung auf Dauer nicht in Erwägung zögen. Dies sei umso bedauerlicher, als dass in den nächsten Jahren mit einem deutlichen Nachwuchsmangel in der Chirurgie zu rechnen sei, der sich jetzt bereits abzuzeichnen beginne $[8,9]$.

Beschäftigt man sich mit diesem drohenden Nachwuchsmangel in den chirurgischen Fächern unter dem speziellen Aspekt der „Feminisierung der Medizin“, besser gesagt der „Frauenpower“ im Medizinstudium, so muss man zwischen genderspezifischen, d.h. alleine die zukünftigen Ärztinnen betreffenden Ursachen und solchen unterscheiden, die den medizinischen Nachwuchs ganz allgemein betreffen. Eine Umfrage von Weber [14] zur Situation von „Ärztinnen in der universitären Chirurgie - Situation im Jahr 2006" nennt in abnehmender prozentualer Bedeutung nachfolgende Gründe für deren Laufbahnhindernisse: $80 \%$ Dominanz der männlichen Strukturen, $70 \%$ fehlendes Mentoring, 67\% zu geringe operative Berücksichtigung, 60\% fehlende Chancengleichheit gegenüber männlichen Ärzten und $49 \%$ geschlechtsspezifische Vorurteile. Bühren [2] fordert angesichts der hierzulande ungenügenden Rahmenbedingungen für die Tätigkeit einer Ärztin 
bei anhaltender Diskreditierung von Teilarbeitsstellen, unzureichenden Angeboten von Serviceleistungen wie der Kinderbetreuung, fehlenden Hilfen bei privater Haushaltsführung und praktisch nicht existenten Erleichterungen beim beruflichen Wiedereinstieg nach einer Schwangerschaft eine Reihe struktureller Maßnahmen, die für einen anhaltenden Attraktivitätsgewinn gerade auch für die chirurgischen Fächer unverzichtbar seien.

In einer Studie von Buxel [5], basierend auf einer 2008/2009 durchgeführten Befragung von 729 Assistenzärzten beiderlei Geschlechts, wurde deren Zufriedenheit mit Arbeitsplatzmerkmalen eruiert. Dabei wurde festgestellt, dass besondere Unzufriedenheit mit dem Stress am Arbeitsplatz, der wenigen Freizeit, den Arbeitszeiten, der Bezahlung sowie dem Freizeitausgleich von Überstunden und nicht zuletzt mit der Ausgestaltung und verlässlichen Umsetzung der Weiterbildung besteht. Die besten Zufriedenheitswerte wurden in dieser Studie bezüglich der Sicherheit des Arbeitsplatzes, der kollegialen Zusammenarbeit, des Klimas im Krankenhaus und zum Standort des Arbeitsplatzes geäußert. Botzlar [1] stellt zu dieser Thematik fest, dass die von werdenden und jungen Ärztinnen und Ärzten vorgefundene Arbeitswelt geprägt sei von kleinteiliger, meist nicht medizinischer Bürokratie bei gleichzeitiger Nichtteilhabe an allen relevanten nicht medizinischen Entscheidungen, von Arbeitsverdichtung als ideenloser Reaktion auf zunehmende Finanzknappheit und von der Frustration der mittelalten ärztlichen Kolleginnen und Kollegen, die vielleicht gerne etwas geändert hätten, sich aufgrund des Zuschnitts ihrer Generation aber nicht getraut haben oder aufgrund der Rahmenbedingungen (Ärzteschwemme!) ohne berufliche Suizidneigung über viele Jahre dazu schlicht nicht in der Lage gewesen sind und nunmehr resigniert hätten. Ähnliche Ergebnisse beschreibt Sauer [13] unter dem Titel „Chirurg - ein Wunschberuf?“, dem die Auswertung einer deutschlandweiten Umfrage unter Studierenden des Jahrgangs 2000/2001 zugrunde liegt. In diesem Artikel wird u. a. darauf hingewiesen, dass sich der Imageverlust des chirurgischen Berufsstands und der negative Einfluss der Medien nicht nur in Auswirkungen auf die Bevölkerung, sondern auch auf den medizinischen Nachwuchs zeige.

Matthes [11] publiziert 2009 unter dem Titel „Ärzte in der Unfallchirurgie - unglücklich und vom Aussterben bedroht?“ das Ergebnis einer Umfrage unter Mitgliedern der DGU, an der sich zu 20\% auch Unfallchirurginnen beteiligt hatten. Der Attraktivitätsverlust der Unfallchirurgie wird dabei auf eine ständig gestiegene Arbeitsbelastung, ungünstige Arbeitszeiten und einen unzureichenden Ausgleich für Bereitschaftsdienste zurückgeführt. Gleiches gelte für unter steigendem ökonomischem Druck entstandene Probleme wie Personalmangel, fehlende Leistungsanreize und eine schlechte Bezahlung. Ungeachtet dessen würden $78 \%$ der Befragten wieder den Arztberuf ergreifen, 85\% gar dasselbe Fachgebiet wählen. Dies deute auf eine Sicherung der inhaltlichen $\mathrm{Zu}-$ kunft der Unfallchirurgie hin, alleine die Rahmenbedingungen müssten geändert werden. Diesem Thema widmet sich auch das gemeinsame Junge Forum von Orthopädie und Unfallchirurgie, in dem Witte und Wölfel [16] unter dem Titel „ein Netzwerk für den orthopädisch-unfallchirurgischen Nachwuchs in Deutschland“ Arbeitsschwerpunkte und Themen formulieren, die zu einer Verbesserung der Situation von Weiterbildungsassistenten/-assistentinnen in Orthopädie und Unfallchirurgie beitragen sollen.

Was muss also angesichts dieser Bestandsaufnahme getan werden, um einerseits dem hohen Frauenanteil in der Medizin gerecht zu werden und andererseits deren während des Studiums offenbar durchaus vorhandenes Interesse an unserem Fachgebiet nachhaltig zu fördern? Neben entsprechender Motivation durch die Lehrenden an den Universitäten, speziell während des Praktischen Jahres, müssen familienfreundliche Strukturen mit Möglichkeiten der Kinderbetreuung (z.B. BG-Klinik Murnau), attraktiven Teilzeitangeboten und Erleichterungen beim beruflichen Wiedereinstieg geschaffen werden. Diskriminierende Äußerungen oder Verhaltensweisen, die von weiblichen Studierenden bzw. Chirurginnen immer wieder thematisiert werden, dürften auch in einem männerdominierten Tätigkeitsfeld längst keinen Platz mehr haben. Auf der Wunschliste junger Chirurginnen ganz oben stehen zudem die Einrichtung eines Mentoring-Programms bei der Karriereplanung (z. B. FiT - Frauen in der Thoraxchirurgie) und eine zunehmende Zahl von Frauen in Leitungspositionen aller chirurgischer Fächer, die ihrerseits eine Vorbildfunktion ausüben können. Nach Haas und Tempka [6] dürfte ergänzend zu der Feststellung, dass die Medizin weiblich wird, für die kommenden 20 Jahre angenommen werden, dass auch die Unfallchirurgie zunehmend weiblich wird. Erste zarte Ansätze in diese Richtung sind bereits zu erkennen, denn es gibt keinerlei Gründe für die Annahme, dass Frauen, die ja bekanntlich auch Marathon laufen oder sämtliche Achttausender besteigen können, nicht auch in einem operativen Fach wie der Unfallchirurgie und Orthopädie hochkompetent tätig sein sollten.

Was zur Nachwuchsgewinnung in unserem Fachgebiet ganz allgemein getan werden muss, ist in einem früheren Editorial bereits umfassend dargestellt worden [15]. Die dort aufgelisteten Forderungen bezüglich Weiterbildung, Arbeitsplatzbeschaffenheit, Motivation und Anerkennung, Entlohnung und Entbürokratisierung sowie im Hinblick auf die Work-Life-Balance gelten geschlechtsunabhängig. Halten wir also fest, dass es eine „Feminisierung der Medizin“ schlichtweg nicht gibt und dass dieser Terminus schon alleine im Sprachgebrauch absolut ungeeignet ist, dass vielmehr dem unbestrittenen nummerischen Überwiegen weiblicher Studierender die erforderliche Beachtung geschenkt werden muss und dass deren Motivation im Hinblick auf das Ergreifen wie auch das befriedigende Ausüben eines chirurgischen Faches, in Sonderheit der Unfallchirurgie und Orthopädie, höchste Priorität einzuräumen ist. Um auf das eingangs zitierte Vortragsthema von Bühren zurückzukommen, ist es unabdingbar, Ärztinnen gerade auch in den chirurgischen Fächern nicht als exotische Lückenbüßerinnen anzusehen, sondern diese vielmehr als willkommenes, eine erfreuliche Zukunftsperspektive bietendes Potenzial erfolgreich tätiger Mitarbeiterinnen und Kolleginnen wertzuschätzen.

\section{K. Weise}

F. Niethard

\section{Literaturverzeichnis}

1 Botzlar A. Motivationskrise junger Ärztinnen und Ärzte. Deutsche Gesellschaft für Chirurgie - Mitteilungen 2009: 329-332

2 Bühren A. Ärztinnen: Zukunftsperspektiven für die Chirurgie. Der Chirurg BDC 2008; 47: 48-50

3 Bühren A. Ärztinnen: Sündenböcke, Lückenbüßerinnen - oder doch die Zukunftsperspektive für die Medizin. Vortrag beim 31. Wissenschaftlichen Kongress des DÄB 11.09.2009, Leipzig

4 Bühren A. Ärztinnen - mehr als nur Lückenbüßerinnen. Deutsches Ärzteblatt 2009; 106: A20-A52

5 Buxel $\mathrm{H}$. Wie gewinnt und bindet man Assistenzärzte leichter? Der Chirurg BDC 2009; 48: 636-640

6 Haas NP, Tempka A. Entwicklung der Unfallchirurgie in den nächsten 20 Jahren. Der Chirurg 2009; 80: 1106-1110

7 Leschber G. FiT - Frauen in der Thoraxchirurgie - wie eine Fachgesellschaft ihren weiblichen Nachwuchs unterstützt. Der Chirurg BDC 2007; 46: $251-252$ 
8 Leschber G. Editorial: Wir haben die Wahl! Der Chirurg BDC 2008; 47: 38-39

9 Leschber G, Ansorg J. Chirurgin in Deutschland: Ergebnisse einer Umfrage 2008. Der Chirurg BDC 2009; 48: 180-187

10 Lindhorst E, Tempka A. Unruhige Zeiten an deutschen Kliniken. Der Unfallchirurg 2005; 108: 1083-1084

11 Matthes G. Ärzte in der Unfallchirurgie - unglücklich und vom Aussterben bedroht? DGU Mitteilungen und Nachrichten 2009; 59: 64-68

12 Nüssler NC. Attraktivitätsverlust der Chirurgie - wie lässt sich gegensteuern? Der Chirurg BDC 2009; 48: 132-133
13 Sauer J, Dommisch K. Chirurg - ein Wunschberuf? Der Chirurg BDC 2006; 45: 145-148

14 Weber T. Ärztinnen in der universitären Chirurgie - Situation im Jahr 2006. Der Chirurg BDC 2006; 45: 367-368

15 Weise K, Niethard FU. Zum Nachwuchsmangel in Unfallchirurgie und Orthopädie. Z Orthop Unfall 2010; 148: 14-17

16 Witte D, Wölfel C. Ein Netzwerk für den orthopädisch-unfallchirurgischen Nachwuchs in Deutschland. Z Orthop Unfall 2008; 146: 298-299 